\title{
THE DERIVATIVE OF A MONOTONIC DISCONTINUOUS FUNCTION
}

\author{
G. PIRANIAN ${ }^{1}$
}

S. Marcus [1] recently showed that if $E$ is a denumerable set of type $G_{\delta}$ on the $x$-axis, then there exists a nondecreasing function $f$ whose derivative is infinite on $E$ and zero outside the closure of $E$. He thus gave a partial answer to a question raised by $Z$. Zahorski $[2$, p. 52, Problem VII]. The present note extends the result of Marcus and thereby completes the solution of Zahorski's problem.

Theorem. If $E$ is a denumerable set of type $G_{\delta}$ on the $x$-axis, then there exists a nondecreasing function $f$ whose derivative satisfies the condition

$$
f^{\prime}(x)= \begin{cases}\infty & (x \in E), \\ 0 & (x \notin E) .\end{cases}
$$

Proof. Let $E=\left\{x_{n}\right\}(n=1,2, \cdots)$ be a set of type $G_{\delta}$. Marcus used the function

$$
f(x)=\sum 2^{-n} f_{n}(x)
$$

where $f_{n}(x)$ takes the values $0,1 / 2$, and 1 on the sets $x<x_{n}, x=x_{n}$, and $x>x_{n}$, respectively. We modify his construction by replacing the coefficients $2^{-n}$ with appropriate values $c_{n}$.

By hypothesis, $\left\{x_{n}\right\}=\cap E_{j}\left(E_{j}\right.$ open, $\left.E_{j} \supset E_{j+1}\right)$. For each $j$, let $\{E(j, k)\}$ denote the family of components of $E_{j}$. Without loss of generality, we may suppose that each $E(j, k)$ has length less than 1 .

Corresponding to the point $x_{1}$ we choose an index pair $\left(j_{1}, k_{1}\right)$ such that $x_{1} \in E\left(j_{1}, k_{1}\right)$; we denote by $d_{1}$ the distance between $x_{1}$ and the complement of $E\left(j_{1}, k_{1}\right)$; and we choose $c_{1}=2^{-1} d_{1}$. After the index pairs $\left(j_{m}, k_{m}\right)$ have been determined for $m=1,2, \cdots, n-1$, we select a pair $\left(j_{n}, k_{n}\right)$ different from all the pairs $\left(j_{m}, k_{m}\right)(1 \leqq m<n)$, in such a way that $x_{n} \in E\left(j_{n}, k_{n}\right)$; and we write $c_{n}=2^{-n} d_{n}$, where $d_{n}$ denotes the distance between $x_{n}$ and the complement of $E\left(j_{n}, k_{n}\right)$. Since each $x_{n}$ lies in infinitely many of the segments $E(j, k)$, the process can be continued without difficulty.

Now let $f(x)=\sum c_{n} f_{n}(x)$. Since $f$ is nondecreasing and has a left-

Received by the editors October 3, 1963.

1 This note was written with support from the National Science Foundation under grant NSF G23830. 
hand and a right-hand discontinuity at each point $x_{n}$, we see immediately that $f^{\prime}(x)=\infty$ at each point of $E$.

On the other hand, let $x \notin E$, let $M$ denote an integer such that $x \notin E\left(j_{n}, k_{n}\right)$ when $n>M$, and let $u \neq 0$. Clearly,

$$
0 \leqq \frac{f(x+u)-f(x)}{u} \leqq|u|^{-1} \sum^{*} c_{n},
$$

where the sum $\sum^{*}$ extends over the terms with indices $n$ for which

$$
x-|u| \leqq x_{n} \leqq x+|u| \text {. }
$$

If $N$ denotes the least of these indices, then $N \rightarrow \infty$ as $u \rightarrow 0$. For all $N>M$, the terms $c_{n}$ that enter the sum $\sum^{*}$ satisfy the condition $c_{n}=2^{-n} d_{n} \leqq 2^{-n}|u|$. Hence the difference quotient is less than $2^{1-N}$, and hence $f^{\prime}(x)=0$. This completes the proof.

\section{REFERENCES}

1. Solomon Marcus, Sur un probleme de Z. Zahorski concernant les points oi la derivée est infinie, Atti Accad. Naz. Lincei Rend. Cl. Sci. Fis. Mat. Natur. (8) 29 (1960), 176-180.

2. Z. Zahorski, Sur la première dérivée, Trans. Amer. Math. Soc. 69 (1950), 1-54.

UNIVERSITY OF MICHIGAN 